\title{
Toward Assembly of Non-close-packed Colloidal Structures from Anisotropic Pentamer Particles
}

\author{
Michael J. Solomon, ${ }^{*}$ Ramsey Zeitoun, Daniel Ortiz, Kyung Eun Sung, \\ Di Deng, Aayush Shah, Mark A. Burns, Sharon C. Glotzer, Joanna \\ M. Millunchick
}

Particles combining multiple anisotropy dimensions offer possibilities for self-assembly that have not been extensively explored to date. The scope for assembly of microparticles in which the anisotropy dimensions of internal bond angle and chemical ordering have been varied is investigated. Colloidal assemblies with interesting open (i.e., non-close-packed) structures can be assembled from these building blocks. The structure of the assemblies formed is linked to the building block anisotropy because the steric constraints introduced induce deviations from close packing. Key challenges addressed in pursuit of these structures are parallelization of microfluidic synthesis methods, simulation to efficiently search the available anisotropy space, and methods that characterize the properties of the resulting assemblies. This combined program of synthesis, simulation, assembly, and characterization may be applied to develop design rules that guide efforts to fabricate microparticle building blocks and their assemblies.

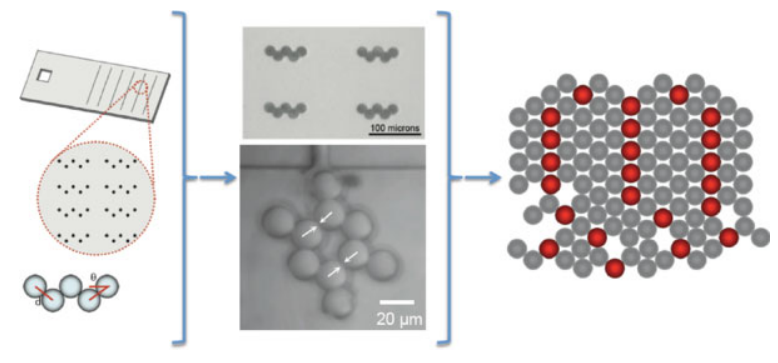

M. J. Solomon, R. Zeitoun, K. E. Sung, M. A. Burns, S. C. Glotzer Department of Chemical Engineering, University of Michigan, Ann Arbor, Michigan, USA

Fax: +1 734763 0459; E-mail: mjsolo@umich.edu D. Ortiz, D. Deng, A. Shah, S. C. Glotzer, J. M. Millunchick Department of Materials Science and Engineering, University of Michigan, Ann Arbor, Michigan, USA

M. J. Solomon, R. Zeitoun, D. Ortiz, K. E. Sung, D. Deng, A. Shah, M. A. Burns, S. C. Glotzer, J. M. Millunchick

Program in Macromolecular Science and Engineering, University of Michigan, Ann Arbor, Michigan, USA

E-mail: mjsolo@umich.edu

M. A. Burns

Department of Biomedical Engineering, University of Michigan,

Ann Arbor, Michigan, USA

\section{Introduction}

The application of anisotropic nanocolloids and microparticles to assemble phases with complex symmetry is a promising approach to create new functional materials. The isotropy of spheres and the centrosymmetric potentials that arise from pair interactions between them yield simple units cells such as face-centered cubic (FCC), hexagonal close-packed (HCP), and body-centered cubic $(\mathrm{BCC}) .^{[1]}$ Mixtures of different size spheres or introduction of dissimilar electrostatic interactions produce other structures such as ionic crystals or quasicrystals. ${ }^{[2-4]}$ For hard particles, introduction of anisometry, as parameterized by aspect ratio, expands the scope for assembly to include liquid crystalline phases such as nematics and smectics. ${ }^{[5]}$ Yet, other material platforms, such as surfactants, block copolymers, and other amphiphiles, yield even more complex phases with useful symmetries due to hetero- 
A)

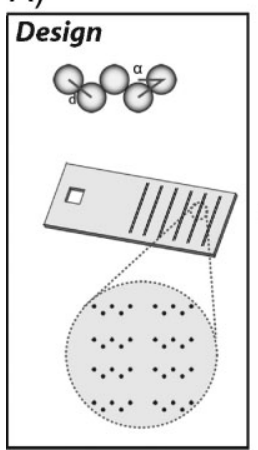

B)

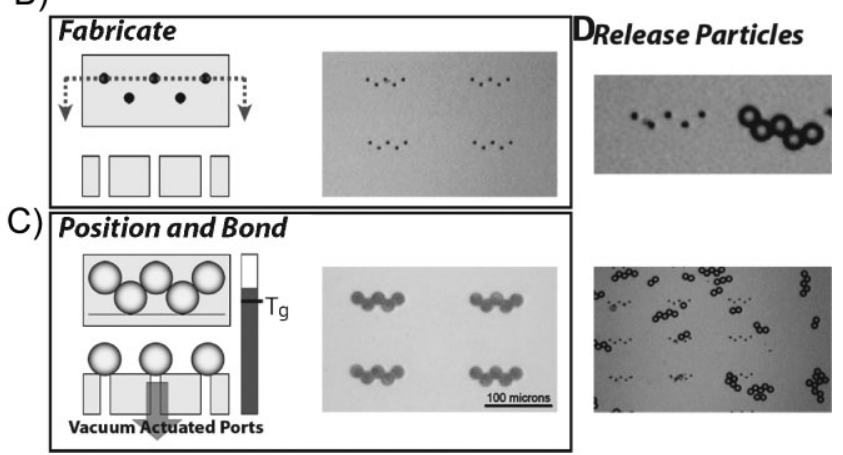

Figure 1. Parallel microfluidic synthesis of pentamer colloidal particles: A) Pentamer particles are designed by specifying bond angles and interparticle distances. These designs are integrated into a massive array of vacuum-actuated holes to position particles. B) Using glass and silicon microfabrication technologies, the array design is generated. C) Particles dispersed in a liquid suspension are positioned onto the holes by fluid flow. The system is drained of liquid and then heated above the glass transition temperature to fuse the particles. D) Particles are sonicated and released from the silicon substrate.

produce a structure indistinguishable from a close-packed layer of spheres. This case of $\alpha=30^{\circ}$ is thus not a particularly interesting target for assembly, since only a simple unit cell results and such an assembly would be readily achievable with a simpler, less elaborate building block. However, for $\alpha \neq 30^{\circ}$ very different assemblies might be achieved, since adjacent pentamers are sterically prohibited from fully interdigitating into a close-packed layer. In this work, we produce particles such as Figure 1A, predict via simulation their two-dimensional (2D) assemblies, and assess if their structure is sufficiently precise so as to offer the possibility of assembly as predicted by simulation. geneity in both geometry and interactions. ${ }^{[6]}$ Assembly of such complex phases is a current goal of colloidal engineering because of possible applications of such ordered arrays in optical and conductive materials as well as in substrates for separations. Combining different kinds of anisotropy, conceptualized as dimensions, in the same particle potentially offers one route to achieve such complex phases. ${ }^{[7]}$ Here we pursue this hypothesis by investigating a particular kind of building block that combines both shape and interaction anisotropy in the same microparticle. We show how a combined program of synthesis, simulation, assembly, and characterization can be applied to develop design rules that can guide future efforts to fabricate useful microparticle building blocks and their assemblies.

The particular class of building block for assembly we investigate is shown schematically in Figure 1A. The particle is comprised of spheres, each permanently bonded into a rigid pentamer with repeating internal structure. Two different dimensions of anisotropy can be varied in this particle class. First, the angle, $\alpha$, which defines the deviation of the five spheres from a linear array, can be varied (cf. Figure $1 \mathrm{~A}$ for definition of $\alpha$ ). Second, varying the composition of the spheres incorporated into the pentamer introduces amphiphile-like anisotropy due to chemical ordering. Particles such as the one shown in Figure $1 \mathrm{~A}$ have been synthesized in small quantities by the microfluidic method of ref. ${ }^{[8]}$ We report here that the effect of varying bond angle in these particles is of particular interest for assembly. To identify possibilities, note that if the internal bond angle is $\alpha=30^{\circ}$, then simple inspection of two aligned pentamers shows that they interdigitate exactly. In this case, a bulk assembly of such pentamers (all comprised of spheres of identical composition) would

\section{Experimental Part Materials}

Particles used were $20 \mu \mathrm{m}$ polystyrene spheres (Precision Size Standard, Polysciences, Inc., $T_{\mathrm{g}}=95^{\circ} \mathrm{C}$ ). Fluids used for particle transport include both $\mathrm{DI} \mathrm{H}_{2} \mathrm{O}$ and dimethyl sulfoxide (DMSO). Fluid flow was actuated using both house vacuum and pressure sources controlled with custom LabVIEW program, VSO-EP pressure controllers (Parker), and a solenoid valve array.

\section{Microfluidic Device Fabrication}

Microfluidic devices were fabricated in both $550 \mu \mathrm{m}$ thick silicon wafers and borofloat glass wafers. Lithography was performed as follows. HMDS microprimer followed by SC1827 photoresist were each spin-coated on the wafer at a $0.5 \mathrm{~K} \mathrm{rpm}$ spread for $4 \mathrm{~s}$ and $3 \mathrm{~K}$ rpm spin for $30 \mathrm{~s}$. Photoresist was exposed using a mask and MA/ BA-6 mask aligner for $13 \mathrm{~s}$ under hard contact mode (Süss MicroTec). Photoresist was developed using MF-319 photoresist developer for $60 \mathrm{~s}$. Silicon features were etched using a deep reactive ion etcher (Surface Technology Systems). Glass was etched by evaporating $500 \AA \mathrm{Cr}$ followed by $3500 \AA \mathrm{Au}$. The same lithography procedure was followed for silicon wafers. Metal layers were etched using Baker gold etchant for $180 \mathrm{~s}$ follow by CR14 chrome etchant for $120 \mathrm{~s}$. Glass features were subsequently etched in $49 \%$ hydrofluoric acid solution until a desired depth was reached. Glass and silicon layers were bonded by either an anodic bond using an SB-6E anodic bonder (Süss MicroTec) or glued with Norland 61 optical adhesive (Norland Products). Individual devices were diced and released prior to operation.

\section{Simulation by Bottom-Up Building Block Assembly}

We use simulation to predict low energy packings of homogeneous and heterogeneous pentamers as motivation for their synthesis 
and assembly. We recently demonstrated a new method for predicting ideal packings achievable through directed assembly and for which the propensity for self-assembly can be determined through comparison with traditional thermodynamic simulation methods such as Monte Carlo or molecular dynamics. ${ }^{[9]}$ Unlike traditional self-assembly methods, BUBBA (Bottom-Up Building Block Assembly) is a heuristic optimization algorithm. The algorithm serves to hierarchically optimize the minimum potential energy of a structure comprised of $N$ building blocks. Although it can, in principle, be used to predict minimum free energy, rather than minimum energy, structures, it is best suited for strongly interacting building blocks where entropy may be neglected, as with heterogeneous pentamers.

Here we apply BUBBA to homogeneous and heterogeneous pentamers configured with uniform internal bond angles. The interaction between non-charged, or between charged and noncharged, spheres on different pentamers is modeled by sticky hard spheres of interaction strength $\varepsilon=1$ in units of $k_{\mathrm{B}} T$ when the spheres are separated by a distance of 0.1 particle diameters or less, and zero otherwise. The interaction between like-charged subunits, which is treated as highly screened, is modeled as an infinitely strong repulsion on contact, and no interaction otherwise. The simulation begins with one pentamer. At each level $N$ of the algorithm, BUBBA builds a sampling of cluster pairs of size $n$ and $m$ where $n+m=N$, which are combined to form candidate minimum energy structures. Each cluster pair is either a previously optimized BUBBA result, or the original building block. To increase the efficiency of the algorithm, we use an angularly discretized interaction potential around both pairs. An intelligent discretization culls combinations of clusters that would result in geometric overlap. Furthermore, at each discrete point, each pair is exhaustively rotated to form even more candidate structures. The relative global energy minima at each step are found by searching all candidate minimum energy structures formed using the above procedure. Therefore, rigorous discretization of the potential results in more accurate results. Since the sampling of cluster pairs can become prohibitively large, BUBBA is an algorithm that is ideal for packings of building blocks with short-range potentials. Here we employ an approximation wherein only the lowest energy clusters of size $n$ and $m$ are used to build a larger cluster of size $N$. Using another model system where shape and interactions were also varied, we found that in one case out of 25 it was necessary to use second-lowest energy clusters of size $n$ and $m$ to obtain the lowest energy cluster of size $N{ }^{[9]}$ For building blocks whose preferred lowest energy packings are crystalline, it is straightforward (although potentially computationally expensive) to carry out the algorithm for larger and larger Nuntil a "converged" pattern is obtained that represents the expected structure in the thermodynamic limit. For non-crystallographic or disordered packings, the structure can depend on $N$, although not necessarily in a statistically significant way.

\section{Results and Discussion}

\section{Anisotropic Particle Synthesis}

Anisotropic particles were previously generated from spherical particle precursors through a microfluidic con- finement technique. ${ }^{[8,10]}$ This method convectively positioned polymeric particles into a channel. Packing under confinement produced repeatable patterns that were a function of particle size, channel width, and channel height. After particles were positioned, structure and anisotropy was preserved by thermally bonding the constituent spheres of the particle. Since the method was performed in a microfluidic system, fabricated heaters quickly raised the temperature of a localized region of the device. Particles were bonded in under $1 \mathrm{~s}$ and fluid flows positioned particles in comparable times. Multifunctional "A-B-A" type particles were synthesized by adding supplemental control lines. Control lines either removed unwanted particles with pressure or positioned desired particles with vacuum.

The microfluidic particle bonding technique provided a first step toward actively creating a variety of particles with designed and programmable anisotropy. However, the system was designed to generate just one particle per fusing cycle. For particle generation to contribute to fundamental studies of anisotropic assembly, a larger quantity of uniform particles must be generated. For example, for pentamers of $20 \mu \mathrm{m}$ particles, 2D assembly over a $1 \mathrm{~mm}^{2}$ surface would require at least 500 particles and for 3D assembly in $1 \mathrm{~mm}^{3}$ at least $\approx 10^{4}$ particles would be required. The number of pentamers required would increase as the constituent sphere size was reduced. Incorporating parallelization into the synthesis so as to generate sufficient particles is thus essential. Parallelization requires the integration of multiple systems to simultaneously position particles in precise configurations. This integration must address technical challenges such as non-uniformities in flow in the microfluidic device as well as increased complexity in design.

To address these needs, we modified the synthetic approach of ref. ${ }^{[8]}$ to achieve parallelization. As an alternative to the original method of positioning particles based on confinement in a microfabricated channel, $20 \mu \mathrm{m}$ particles were positioned on $5 \mu \mathrm{m}$ vacuum-actuated holes (drains). One advantage of these drains is they can be patterned in any shape, thus producing particles that are a direct consequence of the drain patterning on the device, rather than an indirect consequence of channel packing (Figure 1). Furthermore, the number of drains on the substrate can be increased in a straightforward way. Therefore, once an anisotropic particle has been designed, such as the pentamer in Figure 1A, particle patterns can be fabricated (Figure 1B) as per the microfabrication techniques discussed in the Methods Section. These patterns can then be used to align and thermally fuse precursor particles into stable anisotropic shapes (Figure $1 C$ and D). Given the area occupied by a single pentamer configuration in the design $\left(100 \times 200 \mu \mathrm{m}^{2}\right)$, about $5 \times 10^{3}$ particles $/ \mathrm{cm}^{2}$ can be positioned per synthesis cycle, thereby yielding the 
quantities of material necessary for 2D assembly. The pentamer yield is a function of bonding conditions that involves the particle proximity, the bonding temperature, and the method of release.

\section{Simulation of 2D Pentamer Particle Assembly}

The microfluidic synthesis method offers sensitive control of the pentamer bond angle and chemical order. ${ }^{[8]}$ However, the scope to harness such control so as to select specific, optimal building blocks for assembly has not yet been explored. BUBBA simulations find that the bond angle of the homogenous pentamer controls the overall and local packing, and the number, size, and distribution of defects (pores) in the assembled structure. For homogenous pentamers, geometric effects alone dominate their packing. We see from the simulations that the bond angle controls the degree of packing frustration in a structure that, by virtue of the sphere-sphere interactions only, would prefer to be densely packed (Figure $2 \mathrm{~A}-\mathrm{C}$ ). The bond angles $\alpha=0$
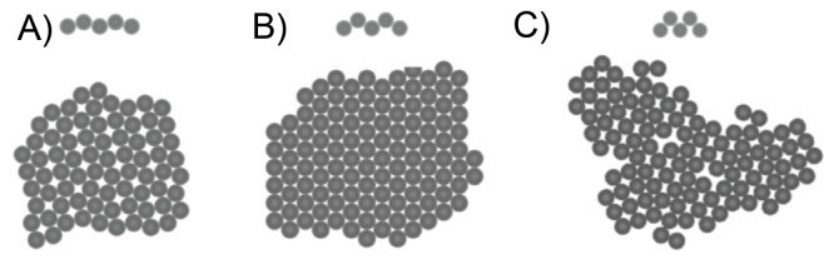

D)

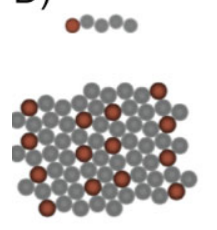

E)

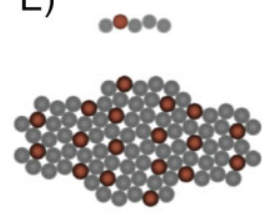

F)

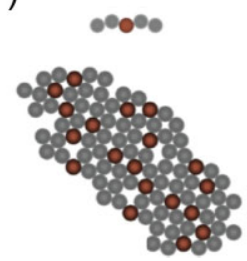

and $30^{\circ}$ (Figure 2B), and $\alpha=60^{\circ}$ are special because they allow in two dimensions perfect HCP packing of the subunit spheres. Intermediate angles (Figure 2A and C) exhibit some degree of packing frustration, resulting in disordered packings that deviate from the optimal HCP structure with noticeable defects (Figure 2). The variation in packing of the structures with bond angle shows the importance of this anisotropy dimension on ideal assemblies.

The presence of one strongly screened charged sphere in a pentamer affects the packing by disfavoring configurations in which charged spheres are touching. The minimum energy packing solutions generated by BUBBA depend on the charge sequence, which can introduce additional frustration depending on the position of the charge for a given bond angle. Figure 2D-F shows the impact of charge sequence on packings of pentamers with $\alpha=15^{\circ}$. The results show that the least perturbative effect on the overall packing relative to $\mathrm{HCP}$ is for the case of the charged sphere in the end position. When the charged sphere moves to the second position, an anisotropy develops in the overall packing to accommodate the frustration in the local packing. The greatest frustration occurs for the charged sphere in the middle position, causing increased anisotropy in the overall packing and increased disorder within the structure with respect to the number, size, and distribution of defects, as well as the distribution of the charged spheres. With this simple example, we see how the additional local packing constraints introduced by charge can be used to affect both the overall packing and the local microstructure.

\section{Potential for Interdigitated Assembly from Pair Binding Studies}

The simulations show that changing the bond angle, $\alpha$, of the anisotropic particles impacts $2 \mathrm{D}$ assembly by affecting how the pentamers pack (see Simulation of 2D Pentamer Particle Assembly Section). For $\alpha \neq 30^{\circ}$ the tendency for the linked spheres to form close-packed structures is frustrated by their connectivity and steric constraints. Patterns with unusual open structures result. Experimental realization of structures such as those shown in Figure 2 requires a collection of particles with tight bounds on the distribution of bond angles. (All the simulated particles are identical; however, the synthesis method will inevitably yield some variation in bond angle, see Anisotropic Particle Synthesis Section.) To test the possible effect of this experimental variability, we introduced pairs of microparticles into a fluidic reservoir and subjected them to mixing flow. ${ }^{[11]}$ The following observations were recorded. First, low bond angle $\left(\alpha<30^{\circ}\right)$ particles preferentially aligned and stably bonded (Figure 3A). Particles with higher bond angles were more likely to disassociate in the flow (Figure 3B). As shown in Figure $3 \mathrm{C}$, the binding was the result of multiple contacts 

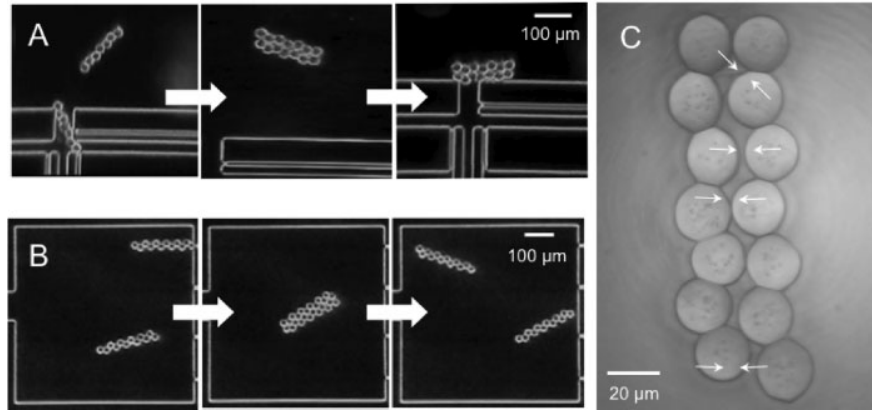

Figure 3. Pair binding of anisotropic particles: A) Pairs of chains (heptamers in this case) with low bond angles $\left(\alpha=12.5^{\circ}\right.$ and $\left.14.9^{\circ}\right)$ preferentially contact and stably bind in mixing flow while those with greater bond angles $\left(B, \alpha=34.6^{\circ}\right.$ and $\left.36.5^{\circ}\right)$ contact, bind, but then disassociate in the flow. The interlocking of the chains shown in A results in multiple binding contacts as evidenced by the high resolution image (C). Interdigitated binding occurs for chains of variable bond angle and length as shown for hexamer chains in (D) with $\alpha=26.6^{\circ}$ and $25.9^{\circ}$. Non-interdigitated assemblies $\left(\alpha=32.5^{\circ}\right.$ and $33.2^{\circ}$ ) also can form, as shown for the case of pentamers in $E$.

between spheres along the particle. The contacts occurred even if the bond angle and length were varied (Figure 3D). Note that because the Figure 3 particles were not thermalized (due to their size) the bonded structures shown are a subset of a large range of steady-state possibilities (e.g., Figure 3E).

These pair binding and interlocking studies were performed for particles comprised of a range of particle lengths (sphere number, $N$ ). For example, particles evaluated in Figure 3 were heptamers (Figure $3 \mathrm{~A}-\mathrm{C}$ ), hexamers (Figure 3D), and pentamers (Figure 3E). The potential for interdigitation, as required for consistency with the Figure 2 simulations, was observed in each of these cases, indicating that the microfluidic synthesis methods produce tight bounds on the bond angle for particles such as the ones shown. Sung et al., ${ }^{[8]}$ moreover, report low standard deviation of the bond angles of microfluidically synthesized particles with sphere number up to $N=10$. Furthermore, consistent with the simulations, in these experiments the bond angle $\alpha=30^{\circ}$ (corresponding to a close-packed configuration) delineates between two possibilities: Below this angle chains can interdigitate and produce multiple binding contacts. Above this angle the pockets between alternating spheres are too small for the chains to interlock, thus reducing the number of binding contacts. Here the observed binding is a consequence of the strong dispersion (van der Waals) interactions of the chains. In particular, binding forces for pairs of polystyrene spheres of comparable size in water have been measured to be $1-2 \mathrm{nN} .{ }^{[12]}$ The non-retarded Hamaker constant of the polystyrene/water/ polystyrene and polystyrene/DMSO/polystyrene systems we study here are $\approx 3.8$ and $1.2 k_{\mathrm{B}} T$, respectively. Such significant short-range van der Waals interactions are consistent with the simulation method that yielded the Figure 2 results. Importantly, the tight distribution in bond

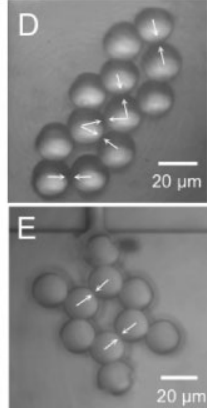

angles both within chains and between chains allows multiple contacts between chains (Figure 3C). Both interdigitated and non-interdigitated bindings are observed, in agreement with the simulations.

\section{Conclusion}

We have introduced rigid pentamers with variable internal bond angles and chemical ordering as particle building blocks from which interesting 2D colloidal assemblies can be produced. Parallelization of microfluidic synthesis methods provides a means to synthesize the $\approx 10^{3}-10^{4}$ particles needed for fundamental studies of 2D assembly. Binding studies of particle pairs show that the bond angles of the particles synthesized are sufficiently uniform that the multiple contacts and interdigitation between particles necessary for assembly are achievable. A simulation algorithm that estimates minimum potential energy structures of the pentamers shows that steric constraints introduced by the internal bond angle of the particles lead to structures that are non-close packed. Varying the chemical ordering of spheres in the pentamers offers additional control of the minimum potential energy structure. The chemical heterogeneity thereby introduced can affect functional properties of the structures. For example, the ability to assemble either dense or porous membranes could yield applications in separations. On the other hand, pentamers comprised of subunits with different conductivities could be used for electronic applications such as colloidal capacitors, batteries, or transistors.

Acknowledgements: The authors were jointly supported in this project by NSF CBET 0707383. RZ also acknowledges NIH T32 EB005582 for support.

Received: August 14, 2009; Revised: November 9, 2009; Published online: December 29, 2009; DOI: 10.1002/marc.200900586

Keywords: anisotropic particles; colloids; computer simulation; microfluidics; self-assembly

[1] W. B. Russel, D. A. Saville, W. R. Schowalter, Colloidal Dispersions, Cambridge University Press, Cambridge 1989.

[2] P. Bartlett, R. H. Ottewill, P. N. Pusey, Phys. Rev. Lett. 1992, 68, 3801.

[3] M. E. Leunissen, C. G. Christova, A. P. Hynninen, C. P. Royall, A. I. Campbell, A. Imhof, M. Dijkstra, R. van Roij, A. van Blaaderen, Nature 2005, 437, 235. 
[4] J. Mikhael, J. Roth, L. Helden, C. Bechinger, Nature 2008, 454, 501.

[5] Z. Dogic, S. Fraden, Curr. Opin. Colloid Interface Sci. 2006, 11 , 47.

[6] F. S. Bates, G. H. Fredrickson, Phys. Today 1999, 52, 32.

[7] S. C. Glotzer, M. J. Solomon, Nat. Mater. 2007, 6, 557.

[8] K. E. Sung, S. A. Vanapalli, D. Mukhija, H. A. McKay, J. M. Millunchick, M. A. Burns, M. J. Solomon, J. Am. Chem. Soc. 2008, 130, 1335.
[9] E. Jankowski, S. C. Glotzer, J. Chem. Phys. 2009, 131, 104104.

[10] S. A. Vanapalli, C. R. Iacovella, K. E. Sung, D. Mukhija, J. M. Millunchick, M. A. Burns, S. C. Glotzer, M. J. Solomon, Langmuir 2008, 24, 3661.

[11] D. Dendukuri, T. A. Hatton, P. S. Doyle, Langmuir 2007, 23 , 4669.

[12] C. S. Hodges, J. A. S. Cleaver, M. Ghadiri, R. Jones, H. M. Pollock, Langmuir 2002, 18, 5741. 